\begin{tabular}{|c|l|}
\hline Title & $\begin{array}{l}\text { Viel Witz, noch mehr Wahrheit. Wie Thomas Brussig die } \\
\text { Vorwendezeit erzählt }\end{array}$ \\
\hline Sub Title & \\
\hline Author & Joch, Markus \\
\hline Publisher & 慶應義塾大学独文学研究室 \\
\hline $\begin{array}{c}\text { Publication } \\
\text { year }\end{array}$ & 2014 \\
\hline Jtitle & 研究年報 (Keio-Germanistik Jahresschrift). No.31 (2014. 3),p.30-54 \\
\hline JaLC DOI & \\
\hline Abstract & \\
\hline Notes & \\
\hline Genre & Departmental Bulletin Paper \\
\hline URL & $\begin{array}{l}\text { https://koara.lib.keio.ac.jp/xoonips/modules/xoonips/detail.php?ko } \\
\text { ara_id=AN1006705X-20140331-0030 }\end{array}$ \\
\hline
\end{tabular}

慶應義塾大学学術情報リポジトリ(KOARA)に掲載されているコンテンツの著作権は、それぞれの著作者、学会または 出版社/発行者に帰属し、その権利は著作権法によって保護されています。引用にあたっては、著作権法を遵守して ご利用ください。

The copyrights of content available on the KeiO Associated Repository of Academic resources (KOARA) belong to the respective authors, academic societies, or publishers/issuers, and these rights are protected by the Japanese Copyright Act. When quoting the content, please follow the Japanese copyright act. 


\section{Viel Witz, noch mehr Wahrheit. Wie Thomas Brussig die Vorwendezeit erzählt}

JOCH, Markus

Im deutschen literarischen Feld nach 1990, besonders unter den Erzählern, dominieren Autoren, die ihre Jugend oder Kindheit noch in der DDR verbrachten, die ersten schriftstellerischen Erfolge dann später, im wiedervereinigten Deutschland erzielten. „Nicht von ungefähr“, befand 2005 Peter Schneider, der westdeutsche Chronist, „haben die Bücher von Ingo Schulze, Thomas Brussig, Jana Hensel, Uwe Tellkamp und vielen anderen eine größere Aufmerksamkeit erfahren als die ihrer gleichaltrigen westdeutschen Kollegen“. Schließlich gelte für die (jüngeren) Schriftsteller aus der vormaligen DDR: „Im Zweifelsfall sind Künstler, für die sich seit dem Fall der Mauer alles geändert hat, produktiver und interessanter als Leute, die sich einbilden, dass für sie alles beim Alten geblieben ist“. ${ }^{1)}$ Der Standortvorteil Ost, von dem hier die Rede ist, lässt sich auf den Begriff des Erfahrungskapitals bringen. Es kennt zwei Gesichtspunkte. „Produktiv“ heißt, dass der lebensgeschichtliche Riss, den die Wende von 1989/90 für die in den 1960er Jahren und später geborenen ostdeutschen Autoren bedeutete, der Trumpf dieser Gruppe war. Weil mit der DDR noch nicht so fest verwachsen wie die ältere Autorengeneration um Christa Wolf, fanden die Jüngeren das neue Deutschland weniger bedrohlich als interessant, was Wahrnehmungsschärfe und Nichtlarmoyanz förderte. Den westdeutschen Altersgenossen wiederum hatte man dreierlei voraus: die Erfahrung des Realsozialismus, einen neugierigeren Blick auf den consumer capitalism, schließlich die Möglichkeit, beide Gesellschaftssysteme

1) Peter Schneider: Wie der Osten gewann. In: Der Spiegel. 2005. H. 48, S. 175. 

aus eigener Erfahrung zu vergleichen - was man konnte, musste man ja auch.

Dennoch, das ,produktiver“ von Schneider ist überzogen. Gegen den Komparativ zu Lasten von Anbietern aus der alten Bundesrepublik spricht das respektable Werk eines Marcel Beyer oder Thomas Hettche, um nur zwei Beispiele für konkurrenzfähige westdeutsche Schriftsteller der 1960er Jahrgänge zu nennen. (Von denen übrigens keiner jemals behauptet hat, für ihn sei nach der Wende „alles beim Alten geblieben“. Man stellte bisweilen fest, dass sich für einen selbst weniger geändert hatte als für die Ostdeutschen - das war alles und ist auch nicht zu leugnen.) Vertretbar dagegen wirkt das ,interessanter“, bezieht es sich doch auf die Wahrnehmung der Leser, professionelle wie nicht-professionelle, auf die Größe, die über Erfolg entscheidet. Dass gelernte Ostdeutsche im Literaturbetrieb um 2000 den Großgewinn in der Ökonomie der Aufmerksamkeit einfuhren, über distinktes Erfahrungskapital nicht nur verfügten, sondern von ihm auch profitierten - was bei der metaphorischen Kapitalsorte so wenig selbstverständlich ist wie bei der buchstäblichen -, verdankte sich neben Qualitäten der Autoren auch dem Erfahrungshorizont der Leser. Inwiefern?

Als Ostdeutscher nach 1990 über die DDR zu schreiben, bedeutete (faktisch, nicht unbedingt intentional), zwei grundverschiedene Nachfragen zu befriedigen: den ehemaligen DDR-Bürgern ein Erinnerungsangebot zu machen, den westdeutschen Rezipienten Exotik zu bieten. Denn für die Mehrheit der Westdeutschen, diejenigen, die zur Zeit des Mauerfalls keine andere als die Nachkriegsordnung kannten, auch keine Ost-Verwandtschaft hatten, war der andere deutsche Staat vor allem eines: fremd. Die Blickverhältnisse zwischen West- und Ostdeutschland vor 1989 hätten asymmetrischer nicht sein können. Während viele DDR-Bürger über kulturelle Entwicklungen im Westen recht gut informiert waren - worüber uns nach der Wende zuvorderst die Romane Brussigs in Kenntnis setzten, ungeachtet ihres Fiktionalitätsstatus aufschlussreicher als jede Reportage -, hatten die meisten Westdeutschen von der DDR so gut wie keine Ahnung. Warum dem so war, plauderte am Vorabend der deutschen Einheit Patrick Süskind aus (Jg. 1949), in 
dankenswerter Offenheit: „[...] Kreta, Andalusien oder die Äußeren Hebriden lagen uns $[\ldots]$ unendlich viel näher als so dubiose Ländereien wie Sachsen, Thüringen, Anhalt, Mecklen- oder Brandenburg, die wir höchstens notgedrungen durchquerten, um auf der Transitstrecke rasch nach Berlin-West zu gelangen. Was hatten wir mit Leipzig, Dresden oder Halle im Sinn? Nichts.“2) Auch deshalb nichts, weil das öffentliche Bekenntnis zur Solidarität mit den Brüdern und Schwestern im Osten, besonders gern vorgebracht am Tag der deutschen Einheit, im Lauf der Jahrzehnte von immer weniger Bundesrepublikanern ernst genommen wurde: „Am 17. Juni gingen wir segeln.“(3) Genau diese Haltung, ein Desinteresse, das nur noch von der Arroganz übertroffen wurde, sollte nach 1989 den ostdeutschen Newcomern zum Vorteil gereichen. Sie hatte zur Folge, dass westdeutsche Leser die DDR nunmehr als ein unbekanntes Land entdecken konnten. „Geschichte aus einem versunkenen Land“, der Untertitel, mit dem Tellkamp 2008 seinen Roman zum Dresden der Vorwendezeit schmückte („Der Turm“), war insofern geschickt gewählt.

Will man die schon länger währende Erfolgsgeschichte Brussigs verstehen, empfiehlt es sich, mit dem Erfahrungskapital zunächst jenes Merkmal zu beachten, das er mit den anderen ostdeutschen Shooting Stars teilt. Innerhalb seiner Autorengruppe aber unterscheidet den Ostberliner das Wie des Erzählens, der Witz. Für sich genommen eine vielfach bemerkte Qualität (Stapel von Seminarund Master-Arbeiten), doch eine, die man in der Literaturwissenschaft nur einmal, und dort auch nur am Rande, als Besonderheit unter den ostdeutschen Anbietern kenntlich gemacht hat. ${ }^{4)}$ Und die Differenz zu westdeutschen Satirikern, Max

2) Patrick Süskind: Deutschland, eine Midlife-crisis. In: Der Spiegel. 1990. H. 38, S. 123.

3) Ebd.

4) Manfred Durzak sieht die Differenz zum nüchternen und verrätselten, leider auch langweiligeren Erzählen von Ingo Schulze: Der Roman zur deutschen Wende? Überlegungen zu Thomas Brussigs Buch Wie es leuchtet. In: Edward Bialek, Monika Wolting (Hrsg.): Kontinuitäten, Brüche, Kontroversen. Deutsche Literatur nach dem 
Viel Witz, noch mehr Wahrheit. Wie Thomas Brussig die Vorwendezeit erzählt

Goldt oder Wiglaf Droste? Ihnen hat der Ostkollege schon das Erfahrungskapital voraus. In einer ersten, einfachen Positionsbestimmung könnte man sagen, dass es die Kombination von Witz und Erfahrungskapital ist, die Brussigs Distinktion im Ganzen des Nach-89er-Feldes ausmacht. Doch kommt ein weiterer Vorzug hinzu, einer, in dessen Licht sich eine Einordnung als ostdeutscher „Satiriker“ versimpelnd ausnimmt: der hohe Wahrheitswert. Ablesbar ist die Verschmelzung von Witz und Wahrheit bereits an der Art und Weise, wie „Am kürzeren Ende der Sonnenallee“ (1999) westdeutsche Überheblichkeit und Kenntnisarmut zu Vorwendezeiten in Szene setzt.

Micha und Mario, die Ostberliner Protagonisten, direkt an der Mauer wohnend und gepeinigt vom zoologischen Ton, den die westdeutschen Touristengruppen auf ihrer Aussichtsplattform anschlagen (,,Guckt mal, 'n echter Zoni!““), entwickeln eine Retourkutsche für die „,ägliche Demütigung““. ${ }^{5)}$ Sie pflegen zum Spaß auf die Reisebusse zuzulaufen und „Hunger! Hunger!“ zu rufen, ${ }^{6}$ was seine Wirkung auf die Besucher nicht verfehlt. Durch die Elendssimulation fühlen sich die Bus-Touristen in ihren schlimmsten Befürchtungen bestätigt (liest man nicht von Versorgungs-Engpässen in der Zone?!). Noch zugespitzt wird die Komik auf westdeutsche Kosten in der - vorausgegangenen - Drehbuch-Fassung: Auf die Zurufe vom Aussichtsturm (,Willste 'ne Banane?“) reagiert der genervte Micha mit der Drohung, später zu den Grenztruppen zu gehen und „euch“, die Westler, „alle abzuknallen“ - daraufhin leert sich die Plattform schlagartig. Warum zündeten derlei Pointen Ende der neunziger Jahre auch, wenn nicht gerade beim westdeutschen Publikum, lösten sie Gelächter im Kino, ${ }^{7)}$ Heiterkeit bei den Lesern aus?

Mauerfall. Dresden 2012, S. $35 \mathrm{f}$.

5) Thomas Brussig, Am kürzeren Ende der Sonnenallee [1999]. Frankurt/M. 2001, S. 9

6) Ebd., S. 42

7) Bei den zwei Besuchen des „Sonnenallee“-Films in einem Berlin-Neukölner Kino von Berlin-Neukölln, November 1999, ernteten Plattform-, Grenztruppen- und Hunger-Pointe an beiden Abenden die meisten Lacher. 
Erstens natürlich, weil die „Hunger“-Show Klischees vom Osten auf die Spitze trieb und so ridikülisierte. Zweitens, weil der Autor die Wahrnehmung der AltBundesrepublikaner sprachlich zu imitieren wusste, in erlebter Rede wiedergab: „Die Touristen waren schockiert über die Zustände, die hinter dem Eisernen Vorhang herrschten [...] [Herv. MJ]“. ${ }^{8)}$ Drittens konnten sich westdeutsche Rezipienten in der Einfalt der Touristenfiguren wiedererkennen, was die Möglichkeit bot, über sich selbst zu lachen. Viertens und vor allem aber erlebten sämtliche Rezipienten eine symbolische Umkehrung der Machtverhältnisse.

Von der Dominanz der Westdeutschen, der im Film die Blickachse vom westlichen Aussichtsturm oben ins östliche Grenzgebiet unten entspricht, lässt das Drehbuch wenig übrig, die Erzählung noch weniger. Brüchig wird die Vorherrschaft nicht nur, weil auf der Handlungsebene den westdeutschen Verhöhnungen eine ostdeutsche Veralberungs- und Einschüchterungskunst antwortet, sondern auch, weil die westdeutsche Rede im Mund des ostdeutschen Erzählers einen lächerlichen, parodistischen Klang annimmt. Der Einsatz erlebter Westrede ähnelt ein wenig dem subversiven Sprachgebrauch der Kolonisierten im 19. Jahrhundert, wie ihn Homi Bhabha schildert: Nichts verunsicherte die englischen Kolonialherrn mehr, als wenn sich das indische Kolonialvolk „Kultur“, „Freiheit“ und „Fortschritt“, die britischen Legitimationsvokabeln, zu eigen machte. Die Wiederholung der Rede der Herrschenden an ungewohntem Ort verlieh dieser eine unfreiwillige Komik, trug so zu schwindender Herrschaft bei. ${ }^{9)}$ Wenn auch nur punktuell: ein narratives Verfahren von Brussig betreffend, ist die Beschreibung der Westdeutschen als Kolonialmacht, der Ostdeutschen als sich wehrende Kolonisierte (das Sinnbild, das nach der Wende eine steile Karriere erlebte ${ }^{10)}$ bedenkenswert. Symbolisch entmachtet durch das „Sonnenallee“-

8) Brussig: Am kürzeren Ende. 1999. Ebd., S. 42.

9) Homi K. Bhabha: Die Verortung der Kultur. Tübingen: Stauffenburg Verlag 2000, S. 48.

10) Vgl. Andrea Geier: Enteignete Indianer und ausgebeutete Neger. Der 
Projekt wurden Westdeutsche aber zuvorderst in Sachen Erinnerungshoheit. $\mathrm{Ob}$ DDR-Jugendliche jemals auf die Idee einer Hunger-Show gekommen sind, das Verhältnis von Fabel und historischer Wirklichkeit also, war für Westler schwerlich überprüfbar - der Ostberliner ,konnte einem viel erzählen“. Ein Erfahrungsrückstand, den sich Brussig guten Gewissens zu nutzen gemacht haben dürfte, war der Wahrheitswert seines Erinnerns doch unabhängig vom Zutreffen dieses oder jenes historischen Details. Er beruhte auf der mentalitätsgeschichtlichen Tatsache, dass es Spannungen zwischen ,Ossis` und ,Wessis“, in den neunziger Jahren das massenmediale Dauerthema, realiter schon vor dem Mauerfall gab, schon die DDR-Bürger (und nicht nur die linientreuen) westdeutsche Selbstgefälligkeit verdross. Das unschöne Faktum plastisch und komisch in Erinnerung zu rufen, statt diskursiv und gekränkt, so die Sonntagsreden von den Brüdern und Schwestern unterhaltsam Lügen zu strafen, darin, unter anderem, bestand die Erzählmoral. Mit der Pointe, dass Michas Wunsch nach Revanche an den Westlern indirekt in Erfüllung geht - durch den Erfolg seines Autors, der den Verkaufszahlen, der feuilletonistischen Beachtung wie auch der Zahl der Übersetzungen nach die meisten westdeutschen Konkurrenten so weit hinter sich lässt, dass sie sich täglich gedemütigt fühlen dürfen.

Warum die Vereinbarkeit von Unterhaltungs- und Wahrheitswert betonen? Unter den poetologischen Selbstbeschreibungen, mit denen Brussig seine Interviews manchmal anreichert, ist die entscheidende wohl: „Das Moralische ohne das Unterhaltende ist Moralin und langweilig. Das Unterhaltende ohne das Moralische ist belanglos und leer. Je unterhaltender man ist, desto moralischer muss man auch sein, und je moralischer man sein will, desto unterhaltender muss man auch sein.“"11) Aus den (frei nach Kant formulierten) Axiomen, zumal ihrem

Kolonialisierungsdiskurs in der Literatur nach 1990. In: Inge Stephan, Alexandra Tacke (Hrsg.): NachBilder der Wende. Köln et al. 2008, S. 70-83.

11) „Das Buch war ein Wagnis“. Thomas Brussig im Interview mit Susanne Messmer. 
sentenziösen Ton, spricht ein gewisser Überdruss. Auf einem „symbiotische[n] Verhältnis““ ${ }^{12)}$ von Unterhaltung und Moral zu bestehen, heißt, sich gegen die Denkgewohnheit zu wenden, wonach Unterhaltung unweigerlich auf Kosten von Wahrheit geht - und umgekehrt. Dass die Vorstellung, Wahrheit („Moral“, „Ernst“) und Witz stünden einander im Wege, selbst noch nach '89 zur doxa im literarischen Feld zählt, zu jenen bewusstlosen Vorannahmen, stillschweigenden Voraussetzungen, die man für so selbstverständlich hält, dass sie nicht einmal explizit formuliert werden müssen, ${ }^{13)}$ wird an keiner Wirkungsgeschichte deutlicher als an der von Brussig. Er, der die Trennlinie zwischen „Spaßmachern und Ernstmachern“ ${ }^{\text {(14) }}$ durch ständiges Überschreiten in Frage stellt, dadurch den wacheren Teil des Feuilletons seit je begeistert, überfordert den langsameren.

Hartnäckig etwa hält sich das Gerücht, eine witzige Verhandlung der realsozialistischen Diktatur laufe auf ihre Verharmlosung hinaus. Ein Hauch der fixen Idee mischte sich sogar in die Reaktionen auf „Helden wie wir“ (1995), obgleich sie im Ganzen doch einem Triumphzug glichen. Wolf Biermanns Huldigung im „Spiegel“, das Buch sei „ein herzerfrischendes Gelächter“, wie auch seine Begründung: „Brussig schildert die grauenhaft komische Leidensgeschichte eines authentischen DDR-Kretins. Und weil deren Zahl Legion ist, könnte Brussigs Buch zumindest im Osten zum Kultbuch werden“, ${ }^{15)}$ unterstrich wie andere Stimmen der führenden Literaturseiten zwei Stärken. 1. In der Figur des angehenden Stasi-Mitarbeiters Klaus Uhltzscht, zusammengesetzt aus

In: Die Tageszeitung, 25.09.2004.

12) Ebd.

13) Vgl. Pierre Bourdieu: Die Regeln der Kunst. Genese und Struktur des literarischen Feldes, Frankfurt a. M. 1999, S. 127, 268, 305.

14) Robert Gernhardt: Versuch einer Annäherung an eine Feldtheorie der Komik. In: Ders.: Was gibt's denn da zu lachen? Kritik der Komiker, Kritik der Kritiker, Kritik der Komik. Zürich 1988, S. 458.

15) Wolf Biermann: Wenig Wahrheiten und viel Witz. In Der Spiegel. 1996. H. 5, S. 186. 
Viel Witz, noch mehr Wahrheit. Wie Thomas Brussig die Vorwendezeit erzählt Minderwertigkeitskomplexen und grotesker Autoritätsgläubigkeit, wird, zumal wenn man sie, wie hier, aus der Innenperspektive beleuchtet, ein Typus kenntlich, ohne den Diktaturen nicht funktionieren. 2. Brussig seziert, indem er ein Produkt des Realsozialismus lächerlich macht, diesen selbst. Und doch, selbst Biermanns Eloge, ein gewaltiger Prestigegewinn für den Jungautor, da das symbolische Kapital des obersten DDR-Dissidenten das Gewicht des Lobes potenzierte - selbst dieser Ritterschlag also wurde von einem leisen Tadel begleitet. Die Staatssicherheit werde „stark verharmlost“; wer einen Stasi-Mann als Trottel zeichne, ignoriere eines: „Es gab im MfS aber auch mehr als genug hochintelligente und effektive Menschenverächter“, ${ }^{16)}$ den Typus Markus Wolf.

So selbstverständlich dieser Satz zutrifft, so schief ist er als Einwand gegen das Erzählkonzept. Als ob das Karikieren einiger Stasi-Trottel ${ }^{17)}$ bedeute, die Existenz effektiver Menschenverächter zu leugnen. Als müsse, wer von der lächerlichen Seite einer Diktatur spricht, partout auch ihre zynische zeigen, am besten in gleicher Ausführlichkeit. Der an Literatur gestellte Dokumentationsanspruch ist schon dubios genug - warum sollte sie verdoppeln müssen, was Journalismus oder Geschichtswissenschaft leisten können? Zudem haftet das Kritikmuster an einem Realismusbegriff à la Lukács', dem Glauben, Romane könnten die Totalität einer Gesellschaft einfangen, statt nur Ausschnitte. Auch sind die Wertungskriterien nicht ganz stimmig: Hätte sich der Erzähler tatsächlich auf intelligente Zyniker konzentriert, wäre das „herzerfrischende Gelächter“ über einen „authentischen DDR-Kretin“ kaum zu haben gewesen.

Doch wie gesagt, bei Biermann überwiegt bei weitem der Respekt, wie weit, gibt das Resümee zu verstehen: „,wenig Wahrheiten, viel Wahrhaftigkeit und noch mehr Witz““ ${ }^{18)}$ Wirklich heikel ist erst das Verhältnis von Rezensions- und

16) Ebd., S. 187.

17) Vgl. Brussig: Helden. 1995, S. 162-185.

18) Biermann: Wenig Wahrheiten. 1996, S. 186. 
Peritext. ${ }^{19)}$ Der zuständige „Spiegel“"-Redakteur wählt als Titel die griffige Zusammenfassung, streicht aber „viel Wahrhaftigkeit“ - den Teil, der Brussigs Fähigkeit auszeichnet, an einer einzelnen Figur das kollektive Duckmäusertum zu treffen. Was übrig bleibt: „Wenig Wahrheiten und viel Witz“, verzerrt den Tenor, spielt Wahrheits- und Unterhaltungswert gegeneinander aus, wo die Besprechung selbst den Wahrheitsgehalt nur eingeschränkt sah. Problem Nummer zwei betrifft eine verpasste Chance. Wie in den frühen Neunzigern die prominentesten Literaturkritiker (Marcel Reich-Ranicki und Helmuth Karasek bei ihrem Angriff auf Jurek Becker), ${ }^{20)}$ so fixiert sich auch Biermann, wenn auch nur gegen Textende, darauf, ob die Stasi denn auch erschreckend genug gezeichnet sei. Darüber vernachlässigt er die Frage, woraus genau der Witz resultiert, wie er etwa zur literarischen Tradition steht. (Was derjenige, der 1972 Heines „Wintermärchen“ adaptierte, vielleicht am verständigsten hätte diskutieren können.) Heute, fast zwanzig Jahre nach Brussigs Durchbruch, stellt sich die offen gebliebene Frage auch, weil kein anderes Werk der Neunziger einen vergleichbaren Begeisterungssturm auslöste, unter ost- und westdeutschen Kritikern gleichermaßen. „Scheinbar naiv, in Wahrheit hintergründig boshaft“, rühmte die Rezensentin der F.A.Z. Mehr: „,genaue Charakter- und Situationsanalysen, die dank einer guten Portion Übertreibung besonders intensiv einleuchten“. Noch mehr: „Nirgends“ lebe die fiktive Autobiographie des Klaus Uhltzsch ,von plattem Witz, sondern immer von der intelligenten Unverschämtheit und dem treffsicheren Spott der Shakespeareschen Narren“. ${ }^{21)}$

19) Peritexte sind die Paratexte, die einen literarischen Text gewissermaßen einkleiden: Titel, Untertitel, Buchcover. Ich übertrage den Begriff auf literaturkritische Texte, in Variation von Gérard Genette. Paratexte. Das Buch zum Beiwerk des Buches. Frankfurt/M. 2001, S. 12.

20) Vgl. zu diesem Trauerspiel Markus Joch: Jurek Beckers „Amanda herzlos“ im „Literarischen Quartett“ (1992). In Carsten Gansel (Hg.): Rhetorik der Erinnerung Literatur und Gedächtnis in den ,geschlossenen Gesellschaften“ des RealSozialismus. Göttingen 2009, S. 343-368.

21) Sabine Brandt: Bleiche Mutter DDR. In: Frankfurter Allgemeine Zeitung, 10.10.1995. 
Viel Witz, noch mehr Wahrheit. Wie Thomas Brussig die Vorwendezeit erzählt Der größte Jubel seit der „Blechtrommel“, wodurch wurde er ermöglicht? Durch Vorsprünge und Missverständnisse.

Der als so überraschend empfundene Mix aus Präzision, Übertreibung und Witz verdankte sich keiner Schöpfung aus dem Nichts. Er entstand aus differentieller Kreativität, jenem Beherrschen des literarischen Erbes, das zugleich über den Stand der Dinge im literarischen Feld die entscheidenden Schritte hinausgeht. ${ }^{22)}$ Vor Brussig war überhaupt nur Einziger darauf gekommen, aus der Froschperspektive eines willfährigen Stasi-Mannes zu erzählen - Jurek Becker mit der Pförtnerfigur in „Der Fluch der Verwandtschaft“ (1980). Deren Anlage jedoch wird in „Helden wie wir“ radikalisiert. (Nicht unbedingt vorsätzlich, versteht sich. Verschiebt ein Autor die avanciertesten ästhetischen Positionen, bedarf es keiner bewussten Anleihen.) Während Becker Folgsamkeit, Beflissenheit und Ängstlichkeit als hervorstechendste Eigenschaften seines Helden lediglich andeutet, treibt Brussig sie ins Karikatureske. Was, zum Beispiel, Einbrecher für Klaus Uhltzscht so „unheimlich“ macht, ist, „daß sie einbrechen, obwohl Einbrechen gesetzlich verboten ist! Was sind das für Menschen, die sich nicht mal von Gesetzen schrecken lassen! Ich fühle mich schon ertappt, wenn ich ein Verbotsschild überhaupt sehe! “23) Auch können die Leser nun, anders als bei der Pförtnerfigur (einem alten Herrn mit unbekannter Vorgeschichte), die Genese einer Ich-Schwäche verfolgen. Nicht allein, dass bereits Klaus' Vater bei der ,Firma“ arbeitet und den Sohn als Versager behandelt, der Protagonist ist auch noch mit einer gouvernantenhaften Mutter geschlagen, Reinlichkeitsfimmel inklusive. Muttersöhnchen-Story und Namenswitz lassen sich verbinden: „Sehen Sie sich doch nur diesen einsamen Vokal an, der unter der Last der Konsonanten ächzt. Das arme U [in Uhltzscht, MJ] kann schon nicht mehr - aber trotzdem werden ihm weitere Konsonanten aufgeladen. [...] Klaus steht für meine leidenschaftliche Artigkeit (es ist zum Heulen, aber so war's - meine Kindheit war

22) Vgl. Bourdieu: Die Regeln. 1999, S. 385.

23) Brussig: Helden. 1995, S. 35. 
ein Exzeß der Artigkeit) und Uhltzscht für mein Abstrampeln, daß ich jede, aber auch wirklich jede Anstrengung auf mich nahm, um meine Mutter nicht zu enttäuschen. ${ }^{\text {(24) }}$ Dass auch noch der Vorname als Problemzone gilt, verweist auf Lyrics eines deutschen Pop-Songs der späten Siebziger, ${ }^{25)}$ Intertextualität zwischen den Kunstsparten.

Es wird also an eine (bei Stasi-Figuren) seltene Innenperspektive angeknüpft interne Fokalisierung plus homodiegetisches Erzählen $-{ }^{26)}$ doch was Becker noch dezent handhabte, erhält nun einen ganz anderen Drive. Dies auch durch ein Vergnügen am Derben, Bizarren und an der Geschichtslegende, das an den frühen Grass erinnert. Den Onanie-Exzessen von Uhltzscht entsprechen die OstseeSzenen in „Katz und Maus“ (1961). Und wenn Uhltzscht mit Hilfe seines Monsterpenis, zu dem er erst unfallbedingt gekommen ist, am Abend des 9. November 1989 die Öffnung der Berliner Mauer erzwingt - die Grenzer treten eingeschüchtert zur Seite -, bestehen Parallelen zur „Blechtrommel“ insoweit, als a) der kleinwüchsige Oskar Matzerath sein Wachstum auch erst am Ende einer ,welthistorischen Epoche' beginnt - 1945 -, b) auch Oskar sein Organ spektakulär einzusetzen weiß - bei ihm die Stimme, mit der er Fensterglas zersingt -, c) Oskar ebenfalls ins politische Geschehen eingreift, wenn auch etwas weniger folgenreich: mit Hilfe seiner Trommel verwandelt er auf der Mai-Kundgebung der Danziger Nationalsozialisten einen Marsch in einen Walzer. Die Konjunktion „wenn auch“ aber ist bereits Gewäsch - dass in „Helden...“ gleich die Weltgeschichte genital umgeschrieben wird, macht eben die Differenzqualität zur Danziger Trilogie aus. Zwischenfazit: Das Neue der Erzählung von 1995 besteht nicht zum wenigsten darin, Errungenschaften älterer Autoren zu steigern und

24) Ebd., S. 43.

25) „Deine Mutter sagte, Klaus, zieh dir bloß die Schuhe aus“. Auf: Marius MüllerWesternhagen: „Mit Pfefferminz bin ich dein Prinz“ (1978).

26) Der Erzähler sagt nicht mehr, als die Figur weiß und ist Hauptfigur der erzählten Welt. Vgl. Matias Martinez, Michael Scheffel: Einführung in die Erzähltheorie. München 1999, S. 64, 82. 

synergetisch $\mathrm{zu}$ vereinen, eher in einem überbietenden und kumulierenden Verhältnis zur Vor-89er-Literatur als in einem Bruch - wie zum Beweis, dass so genannte Einschnitte, und als solcher gilt das Jahr 1989, sich auch in der Literatur als Rekombination von Altem und Neuem gestalten. ${ }^{27)}$

Ein Bruch vollzieht sich erst durch flexibilisiertes Erzählen. Anders als die Altvorderen steigt Brussig wiederholt aus der Rollenprosa aus. Es stimmt nicht ganz, dass er, die Autor-Person, „,von Anfang bis Ende“ „,mit traumwandlerischer Distanz in der authentischen Erzählerebene seines Protagonisten“ ${ }^{28)}$ bleibe. Was auf einer Strecke von 322 Druckseiten auch weniger traumwandlerisch als sklavisch wäre. Lieber changiert er: Mal lässt er allein Klaus sprechen - dort, wo er ihn vorführt -, mal macht er ihn zum Sprachrohr des Autors. Was man vielfach als Höhepunkt des Romans ausgemacht hat, lebt davon; der Hohn auf Christa Wolf wird durch den gleitenden Stimmwechsel zum Highlight jüngerer deutscher Literaturgeschichte.

Klaus ist es, der die bekannteste Rednerin des 4. November 1989 mit der Eiskunstlauftrainerin Jutta Müller verwechselt, dem Idol seiner Mutter. Schon die Assoziation ist eine Frechheit, da der Realperson Müller das Image des Zwangscharakters anhaftet. ${ }^{29)}$ Auch ist sie, die Assoziation, erzähllogisch wohlmotiviert, denn der seiner Willfährigkeit überdrüssige Klaus will gegen diese ganze Riege strenger Mütter (eine Allegorie der Erziehungsdiktatur) endlich

27) Eine generalisierende, doch auch auf Literaturgeschichte beziehbare Beobachtung von Niklas Luhmann: Das Problem der Epochenbildung und der Evolutionstheorie. In: Hans Ulrich Gumbrecht und Ursula Link-Heer (Hg.): Epochenschwellen und Epochenstrukturen im Diskurs der Literatur- und Sprachhistorie. Frankfurt/M. 1985, S. 20,25 .

28) Biermann: Wenig Wahrheiten. 1996, S. 186f.

29) Vgl. Maik Großekathöfer: Popo rein und lächeln. In Der Spiegel. 2000. H. 6, S. 135: „Unverändert sind Fleiß, Ordnung und Zucht feste Größen im Koordinatensystem der Jutta Müller [...].“ 
rebellieren. Bliebe der Autor jedoch wirklich auf der Ebene seines Protagonisten, könnte er kaum betreiben, was er im Kern betreibt, die sprachzentrierte Erledigung von Wolf. Klaus verwechselt die beiden Damen, am Schriftsteller Brussig aber ist es, durch sein Sprachrohr die Verwechselbarkeit Wolfs zu erklären: „Eine echte Eiskunstlauftrainerinnen-Rede, finden Sie nicht? Diese angestrengte Eleganz, dieses Schwelgen in Passagen, die garantiert eine hohe B-Note abwerfen - und gleichzeitig diese kurzatmige politische Programmatik mit einigen verstolperten, verpatzten oder ausgelassenen Sprüngen, die vom betörten Laienpublikum glatt übersehen werden. ${ }^{(30)}$ So spricht ein Stasi-Trottel? Erstaunlich wortgewandt.

Besonders effektiv wirken Protagonisten- und Autorenstimme zusammen, wenn es um die Würdigung von Wolfs Wirken am Runden Tisch geht: „Aber die schönste Verfassungspräambel hat wieder unsere Christa geschrieben. Wie mein Nachname [...] ist auch diese Präambel ein einziges Uhltzscht, wenn Sie verstehen, was ich meine. Alles, aber auch wirklich alles Edle, Wahre, Hehre, Erbauliche usw. wurde dort hineingestopft, verkettet durch Partizipien, natürlich durch das aufdringlichere, das Partizip I. Ich kenne diesen Stil von meiner Mutter: Für den Badekappenzwang, aber sonst liberal.“31) Die Komik resultiert nicht allein aus der Freude der Leser am Wiedererkennen (vielen dürfte das Partizip I schon beim späten Brecht auf die Nerven gegangen sein), auch nicht allein aus den überraschenden Assoziationen (Sprechweise und Badekappe). Mehr noch gründet sie auf dem Kontrast zwischen dem Anspruch oder Wunsch, mit dem die Rednerin auf dem Alexanderplatz so zuversichtlich anhebt - „Jede revolutionäre Bewegung befreit auch die Sprache ${ }^{\text {، } 32)}$-, und dem Krampf, den sie dann in der mündlichen wie in der Papierform bietet. (Tatsächlich geboten hat: „Ausgehend von den humanistischen Traditionen, zu welchen die besten Frauen und Männer [...], gründend auf der revolutionären Erneuerung [...]“, hieß es in der Präambel..) ${ }^{33)}$ Ein

30) Brussig: Helden. 1995, S. 286.

31) Ebd., S. 308.

32) Ebd., S. 283.

33) Präambel von Christa Wolf zur Verfassung der Deutschen Demokratischen Republik: 
Viel Witz, noch mehr Wahrheit. Wie Thomas Brussig die Vorwendezeit erzählt

Kontrast, der Klaus schon während der Verwechslung missfällt: „Vielleicht kann man von einer Eislauftrainerin auch nur erwarten, daß sie wie eine Eislauftrainerin spricht, aber was hat das dann noch mit befreiter Sprache zu tun?“, 34) und dem schließlich, nach Aufklärung des Missverständnisses, eher Brussig als Klaus ein schönes Bonmot widmet: ,Wer glaubt, die Befreiung der Sprache verträgt sich mit der Formulierung, daß aus Forderungen Rechte, also Pflichten werden, verleiht naturgemäß auch einer Verfassungspräambel den Charme einer Heimordnung. “ ${ }^{35)}$ Wie die Komiktheorie schon vor Längerem feststellte, entsteht Lächerlichkeit, wenn die Handlung (bei Wolf Sprachhandlung) als falsches Mittel die Absicht des Verstandes Lügen straft, wenn das Bestreben und die Lage ihren Widerspruch anschaulich in die Höhe treiben. ${ }^{36)}$

In „Helden wie wir“ bildet der Kontrast zwischen Anspruch und Wirklichkeit eine von mehreren Komikquellen. Er ergänzt die auf der Unwissenheit der Figurenstimme beruhende Ironie - vielleicht, erwägt Klaus einmal, habe Wolf in ihrer Rede die Forderung nach Maueröffnung ,einfach vergessen““37)-, und bildet eine Abwechslung zu den Zoten (Wolfs Debütroman als Erektionstöter). ${ }^{38)}$ Der Kontrast hat Teil an Registerwechseln des Komischen, die konkurrierenden Satirikern zu schaffen machen. Zugleich funktionieren sämtliche Register als Medium historischer Einsicht. Denn alles, was an Wolf lächerlich gemacht wird: das Zaghafte, Bedenkliche, ewig Brave und Mahnende, wird nicht als ein Problem der Einzelperson vorgestellt, sondern, die erste Person Plural im Titel signalisiert es schon, als kollektives Syndrom. „Sie war die Autorin für ein Publikum, das es

http://www.mdr.de/damals/archiv/artikel60728.html [Zugriff 15.12.2013]

34) Ebd., S. 286.

35) Ebd, S. 308.

36) Vgl. Jean Paul: Das Lächerliche und das Erhabene [1804]. In: Texte zur Theorie der Komik. Hg. von Helmut Bachmaier, S. 30f.

37) Brussig: Helden. 1995, S. 306.

38) Vgl. ebd., S. 307. 
nicht fertigbringt, ein Dutzend Grenzsoldaten wegzuschieben“: ${ }^{39)}$ Auch hier ist Klartext vernehmbar, überschreibt der Autor seinem Protagonisten die eigene Sicht die Dinge. Gleiches gilt für die Bemerkung „Wie konnte diese Gesellschaft

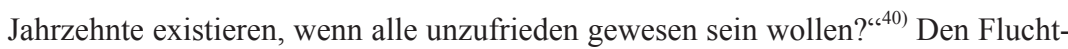
und Nervenpunkt des Romans bildet die kollektive Augenwischerei im Nachhinein.

Deshalb ist es ein Missverständnis zu glauben, Brussig habe in die Bezichtigungsreden, die westdeutsche Literaturkritiker 1989/90 gegen Wolf führten, nur eingestimmt. Ja, er hat sie mit satirischen Mitteln verstärkt, stand den Verächtern sehr viel näher als den Freunden, kam auch aus diesem Grund im westdeutschen Feuilleton an. Und doch wünschte man sich Differenzbestimmungen. Dass die Angreifer des Wendejahres Christa Wolf eine nachträglich vernebelte Nähe zur Staatsmacht nachsagten, mithin Verlogenheit, während Brussig sich über unheilbaren Kleinmut lustig machte, mag man nur für einen graduellen Unterschied halten. Eine Sache aber ist es, wie der Erzähler die Zaghaftigkeit als Kollektivproblem zu verhandeln, und eine ganz andere, nach Art Schirrmachers den Vorwurf der Loyalität zum realsozialistischen Staat auf die Schriftsteller-Elite der DDR zu beschränken. Darin, in der säuberlichen Unterscheidung von wenigen Übeltätern (Partei, Stasi, Partei-Intellektuelle) und Millionen Opfern (alle anderen), in der Weigerung, eine Mitverantwortung - nicht mehr - der vielen auch nur zu erwägen, lag das eigentliche Verharmlosen. Als dessen Korrektiv erweist sich „Helden wie wir“. Was die F.A.Z. 1995 hervorhob, das hintergründig Boshafte, lässt sich gegen den Strich der F.A.Z.-Linie von 1990 lesen, dahingehend, dass ein komischer Erinnerungsmodus der kritischere sein kann, ein ernst auftretender vergleichsweise bequem.

Zum zweiten Missverständnis hat sich der Autor 2004 selbst geäußert.

39) Ebd., S. 316.

40) Ebd., S. 312. 
Taz: „Herr Brussig, Mitte der Neunzigerjahre wurde Ihnen vom deutschen Feuilleton bescheinigt, mit dem Bestseller „Helden wie wir“ den offiziellen Wenderoman geschrieben zu haben.“ Brussig: „Komisch, dass der Verlag dann keinen Aufkleber auf den Umschlag gepappt hat. Andererseits unterstelle ich dem Feuilleton ein krasses Fehlurteil. ,Helden wie wir` beschreibt weder Ursachen noch Verlauf der Wende.“411)

Natürlich war es ein vorteilhaftes Fehlurteil, doch seine nachdrückliche Korrektur leuchtet ein: Die Ursachen der Wende beschreibt der Roman nicht, weil er das Gegenteil erklärt, die langjährige Stabilität der DDR. Den Verlauf der Wende schildert er nicht, da er von der Zeit vor dem 9. November 1989 handelt und mit ihm endet. Die zeitlichen Eckpunkte eines Wenderomans, könnte man fortsetzen, müssen vor und nach dem großen Datum liegen, da Beginn und Vollendung des politischen Umbruchs markieren. Kandidaten für den Anfang im Jahr 1989 wären die ungarische Grenzöffnung, für das Ende 1990 die Währungsunion oder der Tag der Wiedervereinigung. Es gibt ein ganz einfaches Mindestkriterium für den gewünschten Romantyp.

Für manche aber doch zu schwer zu begreifen, und nicht nur in den Neunzigern. Als der Suhrkamp Verlag 2008 dem „Turm“ das falsche Gütesiegel verleiht, obwohl Tellkamp die sieben Jahre vor der Wende schildert, der Unsinn sich allmählich zu einem Running Gag unfreiwilliger Art entwickelt, nimmt Brussig ihn in aufs Korn. In einer Besprechung für den „Spiegel“ bemerkt er am Rande: „,Es ist der lang erwartete große Wenderoman“, heißt es auf dem Umschlag. Dass immer noch und ganz ironiefrei die Erwartung des großen Wenderomans bedient wird, dürfte die literarische Öffentlichkeit ebenso belustigen wie Historiker die Behauptung, dass die Wende am 9. November abgeschlossen war; da endet

41) „Das Buch war ein Wagnis“, 25.09.2004. 
nämlich der Roman [...].“42) Die Zuschreibung als absurd kenntlich zu machen ist niemand berufener als ein Rezensent, dem Gleiches widerfahren ist. Für den bissigen Schlenker gibt es aber noch einen weiteren Grund. Dass besagter Klappentext den lange erwarteten Wenderoman annonciert, muss wie Hohn in den Ohren eines Autors klingen, der das Ersehnte längst geliefert hat - vier Jahre zuvor, mit „Wie es leuchtet“, dem Buch, von dem er im Taz-Interview das 95erWerk abheben muss, um dem Irrtum vorzubeugen, er wiederhole sich. „Wie es leuchtet" hat „Helden... " und „Der Turm“ schon mal voraus, das Mindestkriterium für einen Wenderoman zu erfüllen (und erfüllt noch weit mehr). ${ }^{43)}$ Der Klappentext von Suhrkamp aber tut so, als habe es Brussigs Werk nie gegeben. Insofern ist er nicht nur unfreiwillig komisch, er ist auch ein Akt des Invisibilisierens - auf den der Herausgeforderte geschickt reagiert. Erklärt Brussig kurz, warum „Der Turm“ kein Wenderoman sein kann, und schickt er der Begründung ein „,immer noch“ voraus, lässt er durchblicken, dass und warum es schon einen gegeben hat. Andeuten genügt.

Bei allem Eigeninteresse gibt es auch einen poetischen, letztlich schwerer wiegenden Grund, Vorwende- von Wenderomanen abzusetzen. „Der Turm“, erläutert der Rezensent, ist nicht nur kalendarisch ein Vorwenderoman, er ist es vor allem wegen des realistisch gezeichneten Horizonts seiner Protagonisten: „Er erzählt eine DDR, die von ihrem Ende noch nichts wusste. [...] von ,Endzeit‘ wurde erst hinterher geredet.“ Eine gelungene Erzählung hält die Sicht damaliger Akteure von späterem Wissen frei. In dieser positiven Fremd- scheint mir Selbstbeschreibung zu stecken. Was der Rezensent am Dresdner Kollegen lobt, zeichnet schon seine eigenen Vorwende-Erzählungen aus.

In „Helden...“ ist es das Verhältnis von erinnerndem und erinnerten Ich, dass das Noch-nicht-Wissen zur Geltung bringt. Der sich nach der Wende gegenüber einem

42) Thomas Brussig: „Schau genau hin.“ In: Der Spiegel. 2008. H. 40, S. 153.

43) Vgl. den Aufsatz von Durzak (Anm. 4). 
US-Journalisten erinnernde Klaus - dies die Rahmenerzählung - schüttelt permanent den Kopf über sein willfähriges Vorwende-Ich, das erinnerte Ich, dessen Erlebnisse die Binnenerzählung und damit den Erzählkern bilden (deshalb: Vorwende-Roman). Vor dem 4. November 1989 gibt es in der erzählten Zeit keinen Moment, in dem das erinnerte Ich die Möglichkeit eines Endes der DDR, und damit die Möglichkeit von etwas Renitenz, auch nur erwägen würde. Zusätzlich akzentuiert wird der Glaube der DDR-Bürger, ihr Staat währe ewig oder doch zumindest bis zu ihrem Lebensende (wovon übrigens auch die meisten Westdeutschen ausgingen, wenn sie sich richtig erinnern würden), durch die Figur von Klaus' Mutter. Für sie, die parteifromme, ist der Untergang ihres Staates ebenso undenkbar, anders als beim Sohn aber beruht die Undenkbarkeit bei ihr immer auch auf Selbstgewissheit - der Selbstgewissheit einer selbst- und rastlos für den Arbeiter- und Bauern-Staat tätigen Ärztin. So betonköpfig und lächerlich das Unverständnis wirkt, mit dem sie auf die Alexanderplatz-Demonstration reagiert, es mutet zugleich ,logisch', das heißt vom Autor schlüssig motiviert an: „Ich weiß nicht, was das soll. [...] Wir haben uns für die Menschen aufgeopfert. Für ganz normale Menschen. Deshalb sind wir Helden. [...] Helden wie wir haben nichts zu bereuen. “44)

In „Am kürzeren Ende...“ wird der Horizont der sich „,im Dunklen des gegenwärtigen Augenblicks“ (Bloch) befindlichen DDR-Bewohner noch deutlicher. Dies zunächst durch die interne Fokalisierung, durch einen Erzähler, der nicht mehr sagt und weiß als sein Protagonist: Nicht nur Micha ahnt nichts vom nahenden Ende der DDR - er ist viel zu sehr damit beschäftigt, die schöne Miriam zu erobern -, bis vier Seiten vor Erzählschluss gibt es auch keine Erzählerstimme, die auf das Ende vorausdeutete. Brussig verschont uns mit Prolepsen, großartig. Noch relevanter allerdings sind jene zwei Schlüsselstellen, die erstmals vorausdeuten und dadurch auf einen unvermeidlich engen Horizont aufmerksam machen.

44) Brussig: Helden. 1995, S. 299. 
Gelegenheitsschriftsteller Micha Kuppisch resümiert das alltägliche Treiben in der Sonnenallee, von den ewig erfolglosen Karriere-Ambitionen des AbschnittsBevollmächtigten bis zu den kleinen Anpassungen von Vater Kuppisch - jene Alltags-Aktionen, die alle möglichen Ziele verfolgen, nur keinen ,politischen Widerstand“ -, im Nachhinein mit einer lakonischen Bemerkung: „Es wäre ewig so weitergegangen." Wenige Zeilen danach wiederholt eine andere, die Erzählerstimme diese Bemerkung und erläutert den Irrealis „,wäre“: „Es wäre ewig so weitergegangen, aber es ist was dazwischen kommen. “45) Die Redewendung ,es kommt was dazwischen“ bezeichnet im Deutschen meist Unangenehmes, Unfall oder Krankheit, und immer ein unvorhersehbares Geschehen. In der Erzählung nun ist das „aber es ist etwas dazwischen gekommen“ krasses Understatement. Denn es meint a) statt Negativem Erfreuliches und b) weit mehr als nur Erfreuliches. Es meint etwas, von dem die meisten DDR-Bürger nur träumen konnten. „Dazwischen“ kam der Mauerfall. Die Erzählerstimme ergänzt Micha zwar, aber macht sich nicht klüger als ihn; sie behandelt durch ihre Wortwahl den Mauerfall als etwas, was der Protagonist wie alle anderen Figuren nicht erahnen konnte.

Unvorhersehbares in der hier verhandelten, der höchst erfreulichen Variante nennt die Alltagssprache gern „Wunder“. Davon handelt das Story-Ende (bevor sich die Erzählererstimme noch einmal einschaltet - dazu gleich): Als Marios Existenzialistin in den Wehen liegt und nicht rechtzeitig ins Krankenhaus zu kommen droht, tritt ein als Michail Gorbatschow erkennbarer Russe auf, dem einfach alles gelingt. Er „machte nur eine Handbewegung zum Himmel - und augenblicklich hörte es auf zu regnen“. Er bewährt sich auch als Geburtshelfer: „ein paar Augenblicke später kam er wieder aus dem Auto und hielt ein fertig gewickeltes [!] Neugeborenes, das er Mario in den Arm legte.“ Jetzt müsste er nur noch Marios Auto zum Anspringen bringen. „Nachdem der Russe beide Hände

45) Brussig: Am kürzeren Ende. 1999, S. 153. 
Viel Witz, noch mehr Wahrheit. Wie Thomas Brussig die Vorwendezeit erzählt frei hatte, berührte er die Motorhaube des Trabis. Der Wagen sprang sofort wieder an.“ Dem Hinweis auf etwas Unvorhersehbares folgt mit der Geschichte vom „Wunderrussen“46) also ein Märchen. An dieser Stelle der Erzählung zeugt das Märchen von maximalem Realismus. Ja, Realismus. Denn so irreal das erzählte Geschehen ist, die Bemerkung zur Unvorhersehbarkeit und die direkt anschließende Gorbatschow-Geschichte verweisen, in ihrer Kombination, auf etwas sehr Reales: auf eine kollektive Wahrnehmung. Weil, erstens, die meisten DDR-Bürger die Wende als großes Glück empfanden - zumindest im Wendejahr von 1989/90 - ${ }^{47)}$ aber, zweitens, vor dem Herbst 1989 so gut wie niemand das Ende der DDR vorhersah, nahmen die meisten den für die Wende wesentlich Mitverantwortlichen, Gorbatschow, tatsächlich als einen Wundertäter wahr, nicht weniger. Die unbestreitbar reale Wahrnehmung (real im Sinn von: vorhanden, verbreitet, vorfindlich - ob sie auch richtig war, ist unerheblich) erfasst Brussig mit dem ihm eigenen Mittel der Komik, der irrealen Überspitzung: Gorbatschow als Christus-Figur, die, wenn nötig, auch über Wasser gehen könnte. Vielleicht dient die Überspitzung sogar dazu, sich über die Kollektivwahrnehmung ein wenig lustig zu machen? Dann wäre es sogar ,kritischer Realismus', etwas für die engagierte Deutschlehrerin.

Wie auch immer, das Märchen zeugt von einem Erzählprogramm, das wir Wahrnehmungsrealismus nennen können. Unterscheidbar vom Fakten- oder Oberflächenrealismus, der auf audiovisuell dokumentierbare Realien aus ist, bildet der Wahrnehmungsrealismus, der hellere Bruder, noch in einer anderen Hinsicht Brussigs „Erzeugungsformel“ (unter dem Begriff versteht man das Erzeugungsprinzip eines oder mehrerer Werke, durch das sich ein Schriftsteller von zeitgleichen oder vorausgegangenen Kollegen unterscheidet). ${ }^{48)}$ Der

46) Sämtliche Zitate ebd., S. 156

47) Im Nachhinein wurde sie von einem Teil der Ex-DDR-Bürger, will heißen: von den ökonomischen Verlierern, den arbeitslosen gewordenen, als verheerend eingeschätzt. 48) Vgl. Bourdieu: Die Regeln. 1999, S. 61. 
„Sonnenallee“-Stoff, unterstreicht Brussig in einem Interview von 1999, anlässlich des Erscheinens von Film und Buch, „versucht nicht zu schildern, wie die DDR war, sondern erzählt, wie sie gern erinnert wird.““99) Dieser nicht ganz unwichtigen Bemerkung entspricht der abschließende Kommentar der Erzählerstimme. Er warnt davor, sich der Erinnerung hinzugeben, denn sie vollbringe „beharrlich das Wunder, einen Frieden mit der Vergangenheit zu schließen, in dem sich jeder Groll verflüchtigt und der weiche Schleier der Nostalgie über alles legt, was mal scharf und schneidend empfunden wurde. Glückliche Menschen haben ein schlechtes Gedächtnis und reiche Erinnerungen. ${ }^{\left({ }^{60)}\right.}$ Es geht in Erzählung wie Film erklärtermaßen darum, einen ostalgischen Wahrnehmungsmodus zu zeigen, als solchen kenntlich zu machen, zu objektivieren. Schon angesichts der Selbstkommentare, die eigentlich unüberhörbar sein sollten, wurden sie doch in einem weithin wahrnehmbaren Begleitdiskurs („Spiegel“-Interview) wie auch im Werk selbst platziert, und dort an besonders auffälliger Stelle (als Schluss-Sätze und als Klappentext) - schon angesichts der Selbstkommentare also muten die Vorwürfe gegen „Sonnenallee“, die man in Feuilleton und Germanistik geradezu wütend erhob, abstrus an. „Keinerlei Erinnerungskultur“ und „zurechtgeschönte“ DDR-Vergangenheit? ${ }^{51)}$ „Verdrängung““ des DDR-Unrechts? ${ }^{52)}$ „Verklärung““? ${ }^{53)}$ Wirklich verklären und schönen kann nur, wer dem Publikum weismachen will:

49) Vgl. „Jubelfeiern wird's geben.“ Thomas Brussig im Gespräch mit Volker Hage. In: Der Spiegel. 1999, H. 36, S. 255.,,Und auch der "Sonnenallee"-Stoff geht nicht in die eigene Falle: Er versucht nicht zu schildern, wie die DDR war, sondern erzählt, wie sie gern erinnert wird.“

50) Brussig: Am kürzeren Ende. 1999, S. 157.

51) Ute Stempel: Keinerlei Erinnerungskultur. Thomas Brussigs albern-versöhnliche 'Mauerkomödie' Neue Zürcher Zeitung, 8. Februar 2000.

52) Christiane Peitz: Alle so schön grau hier. In: Die Zeit. 45.1999.

53) Frank Thomas Grub: ,Wende“ und ,Einheit‘ im Spiegel der deutschsprachigen Literatur, Bd. 1. Berlin 2003, S. 570.: „Häufig lässt sich ,Ostalgie“ als Gesamthaltung von Texten erkennen. Ein Beispiel hierfür ist Thomas Brussigs Am kürzeren Ende der Sonnenallee (1999). Die Verklärung des Lebens in der DDR wird zwar bisweilen relativiert, doch insgesamt wirkt der Alltag relativ ,harmlos‘.“ 
So wie ich es schildere, war es. Und, wichtiger: Wer einen verklärenden Blick zurück vorführt, erliegt ihm nicht. Das wären die ersten Einwände.

Wenn Micha, Mario und Wuschel den Mädchen hinterher steigen, viel Motorrad fahren, katastrophal tanzen, für eine wichtige LP eine wahre Odyssee auf sich nehmen, im Drogenrausch das Mobiliar zerlegen usw.; wenn sie also machen, was vernünftige Jugendliche in den Achtzigern auch im Westen machten; und wenn gleichzeitig vom Stasi-Terror wenig die Rede / zu sehen ist, liegt natürlich immer auch eine Selektion der sozialen Wirklichkeit nach Unterhaltungskriterien vor. Nur ist diese Selektionsart erzählmoralisch unproblematisch, ihre Einordnung unter Verklärung, Verdrängung, Beschönigung, und was der Bezichtigungsvokabeln mehr sind, kurzschlüssig. Zu erläutern ist das nicht etwa, weil Brussig der Verteidigung bedürfte. (Wir reden von einem Autor, der für das „Sonnenallee“-Skript 1999 den Deutschen Drehbuch-Preis erhielt, sich allein bis 2003 über 2,6 Millionen Kinozuschauer freuen durfte und mit der Erzählung seinen zweiten Bestseller landete. Und das mit 35. Im Übrigen gab es auch zahlreiche positive Rezensionen. Was neidische Feuilletonisten von seinem Werk halten, dürfte da von überschaubarem Interesse sein.) Nein, auf die „Verdrängungs“- und „Verklärungs“-Rede ist aus einem anderen Grund zu reagieren. Sie hat sich mittlerweile zum Forschungsproblem entwickelt. Zum einen, weil es ein namhafter und ansonsten kenntnisreicher Kollege ist, der die Rede kolportiert. ${ }^{54)}$ Zum anderen, weil sie bei Nachwuchswissenschaftlern Wirkung zeigt. Die nämlich, lässt eine jüngere Publikation erkennen, ${ }^{55)}$ sind vom „Sonnenallee“-Stoff sehr angetan, kennen sich auch bestens in ihm aus, aber sie zeigen sich von der Phalanx der Verklärungsvorwürfe doch eingeschüchtert. So sehr, dass sie bang fragen, ob man denn über Diktaturen auch lachen darf. Und den auf Verklärung abhebenden ,Staatsanwälten“ nur entgegenzuhalten wagen,

54) Vgl. Fußn. 55.

55) Vgl. Rita Bartl u.a. (Hg.): Ostalgie in Gesellschaft und Literatur. „Am kürzeren Ende von Sonnenallee“von Thomas Brussig. Norderstedt 2013, bes. $143 \mathrm{ff}$. 
Buch und Film seien doch eine Komödie, keine Dokumentation. Richtiges Argument, natürlich. Aber das Gattungsargument allein scheint mir arg defensiv. Daher hier kleine Erweiterungen.

Erstens drängt sich die Frage auf, ob die Kritiker das Schlusswort des ,FilmMicha', gesprochen unmittelbar vorm Abspann, überhaupt zur Kenntnis genommen haben - oder im Geiste bereits beim Rotwein waren. Das Schlusswort, lapidar daherkommend, gleichwohl eine Coda, lautete: „Es war einmal ein Land, und ich hab' dort gelebt. Wenn man mich fragt, wie's war: Es war die schönste Zeit meines Lebens. Denn ich war jung - und verliebt. ${ }^{(56)}$ Schön, so kann man ausbuchstabieren, war es nicht, weil das politische System der DDR großartig oder auch nur akzeptabel gewesen wäre. Schön war es, weil ich in meiner Jugend anderes zu tun hatte, als mich um Politik zu kümmern. Es gab da nämlich ein Mädchen. - Wovon Micha (implizit) spricht, nennt man in der Politologie den Unterschied von System und Lebenswelt. Es gibt stets eine Lebenswelt jenseits des jeweiligen politischen Systems, und sich lieber an sie zu erinnern als an Letzteres, ist, wenn man auf die Jugend zurückschaut, nur ,natürlich`. ,Natürlich` aus Gründen des Selbstschutzes. Der „Sonnenallee“-Stoff handelt nicht von der DDR, er handelt von einer zurückliegenden Jugend in der DDR, insoweit ist Nostalgie kaum vermeidlich, mehr, legitim.

Zweitens: Wer das Treiben einer Clique wie der von Micha schildert, dem geht es nicht um die Verharmlosung des Realsozialismus, sondern um den Nachweis, dass man sich als Jugendlicher auch in einer Parteidiktatur Freiheiten verschaffen konnte, darum, sich den mitleidigen Blick westdeutscher Altersgenossen zu verbitten. Dass diese Intention eine vorsätzlich selektive Retrospektive auf die DDR erforderte, ein Anspruch auf realistische ,Gesamtschau' aber auch gar nicht erhoben wurde, macht der Schlusssatz des Buches („Glückliche Menschen haben ein schlechtes Gedächtnis...") transparent genug.

56) Sonnenallee. Ein Leander Haußmann Film. Deutschland 1999. Min. 1:20:28ff. 
Drittens gilt für die Erinnerungen an kleine Fluchten wie die Pop-Musik, für die Selektionen im Ganzen, dass sie kein Iota an der Verachtung für den Realsozialismus ändern, an verächtlichen Wertungssteuerungen, die sich in der Story auf Schritt und Tritt finden. Dass sie komisch formuliert sind, macht sie für die wirklichen, die SED-Nostalgiker umso schmerzhafter. Brille, der Intellektuelle in Michas Clique, erklärt einmal, dass es in der DDR keine unpolitischen Studiengänge gibt. Er ,wußte sogar, daß das Studium der Ur- und Frühgeschichte nicht unpolitisch ist: Da lernt man auch nur, wie die sich schon damals nach der SED gesehnt haben. “57) Die Partei als Avantgarde, die selbst die Träume der Neandertaler erfüllt - treffsicherer kann man mit dem Selbstvertrauen der Historischen Materialisten kaum umgehen. Verachtung produziert man (unter anderem), indem man zeigt, dass zwischen Selbstvertrauen und Realgröße des Objekts eine gewisse Differenz besteht. Lächerlich zu machen versteht Brussig vor allem die erzieherische Seite der Genossen. Als Mario eine Lenin-Parole verballhornt hat („Die Partei ist die Vorhaut der Arbeiterklasse“), verdonnert ihn Staatskunde-Lehrerin Erdmute Löffeling zu einem Diskussionsbeitrag, obwohl derlei Arbeiten doch eigentlich als eine echte Ehre gedacht sind. ${ }^{58)}$ Da entsteht die Komik aus dem performativen Widerspruch zwischen alltäglicher und erträumter Funktion, wie auch daraus, dass der Lehrerin, wenn sie von Strafe spricht, die Wahrheit unterläuft. (Zote und Namenswitz sind auch nicht übel.) Lächerlichmachen, komisch munitionierte Verachtung, ist das Gegenteil von Verklärung. Seltsam, dass man an Banalitäten erinnern muss.

Viertens setzt Brussig ein wirkungsvolles Gegengewicht zur Selektion ein, den jähen Umschlag vom Idyllischen ins Entsetzliche. Mitten in eine putzige Geschichte über DDR-Bürger, die Fremdsprachen lernen, die in Ländern gesprochen werden, in die sie doch gar nicht reisen durften, lässt er eine

57) Brussig: Am kürzeren Ende. 1999, S. 42.

58) Vgl. ebd., S. $21 \mathrm{f}$. 
,Anekdote، zum Stasi-Terror einschlagen. Sie handelt von einem Modelleisenbahn-Liebhaber, dem die Kontakte zu westdeutschen HobbyFreunden eine Verhaftung wegen Agententätigkeit einbringen. „Als Günter nach einem Jahr und acht Monaten zurückkam, benötigte er zum Atmen eine Apparatur, die er auf einem Wägelchen hinter sich her ziehen musste. ${ }^{(59)}$ Noch Fragen?

(慶應義塾大学文学部訪問准教授)

59) Ebd., S. 125. 